\title{
Psychopathology as the basic science of psychiatry
}

\author{
Giovanni Stanghellini and Matthew R. Broome
}

\section{Summary}

We argue that psychopathology, as the discipline that assesses and makes sense of abnormal human subjectivity, should be at the heart of psychiatry. It should be a basic educational prerequisite in the curriculum for mental health professionals and a key element of the shared intellectual identity of clinicians and researchers in this field.

Declaration of interest

None.
Giovanni Stanghellini (pictured) is Professor of Dynamic Psychology and Psychopathology at 'G. d'Annunzio' University (Chieti, Italy) and Profesor Adjuncto 'D. Portales' University (Santiago, Chile). He chairs the World Psychiatric Association Section on Psychiatry and the Humanities, and the Association of European Psychiatrists Section on Philosophy and Psychiatry. Among his most recent books are Emotions and Personhood (with R. Rosfort), One Hundred Years of Karl Jaspers' General Psychopathology (co-edited with T. Fuchs) and the Oxford Handbook of Philosophy and Psychiatry (co-edited with K. W. M. Fulford et al) (all Oxford University Press, 2013). Matthew Broome is a clinical academic in Oxford. His research interests include the prodrome of psychosis, delusions, neuroimaging, mood instabilty, and the philosophy of psychiatry.

Psychopathology ${ }^{1,2}$ is the discipline that provides psychiatrists with basic knowledge about the abnormal phenomena that affect the human mind and with a valid, reliable method to appraise them. In contemporary usage, the term 'psychopathology' is employed in a number of different ways. A common way to use it is to conflate psychopathology with symptomatology - the study of isolated symptoms in view of their diagnostic and aetiological significance. Assessing symptoms allows the identification of specific diagnostic entities that, in turn, enable prediction of natural history and response to treatment. Psychopathology is about that, but not just about that. Whereas symptomatology is strictly illness oriented, psychopathology is also person oriented, since it attempts to describe the patient's experience and her relationship to herself and to the world.

Biomedical science was in part built upon the transformation of a complaint into a symptom. This allowed medical science to see in a complaint (e.g. exhaustion) the effect of a pathological cause active in the human body (e.g. an endocrine dysfunction). This move from complaint to symptom to pathophysiology may overshadow the fact that a complaint has a meaning for the individual sufferer: it expresses a question or desire. A person may not necessarily seek elimination of his complaint, but rather fulfilment of his desire (e.g. to see the doctor fail and himself triumphantly to become an incurable patient). Biomedical science's success can be viewed as due to its focus on how symptoms relate to causes and treatment; this success is due to partial exclusion of the subjectivity of the patient and the meaning their symptoms hold for them. A psychopathological approach, by contrast, does not exclude seeing abnormal phenomena as symptoms caused by a dysfunction to be cured, but additionally includes the exploration of personal meanings. Psychopathology focuses on the experiencing subject and can operate in parallel with a traditional biomedical approach. The patient is an active partner in the diagnostic process, capable of interpreting her own complaints. Symptoms are conceptualised as the outcome of a mediation between a vulnerable self and the sick person trying to cope with and make sense of her complaints. ${ }^{3}$

A second use of the term psychopathology is as a synonym to nosography. The latter term outlines conventional characteristics of a syndrome (i.e. a combination of symptoms empirically aggregated) and thereby serves the goal of a clinical diagnosis. Psychopathology is not merely about diagnosis. In psychopathology, what matters most is that the 'chaos of phenomena' should stand out in an evident way and in multiple connections. Psychopathology promotes attention to the person's whole field of experience, rather than a restricted focus on symptoms selected according to their putative diagnostic relevance. The existing classifications of mental illnesses are merely provisional diagnostic conventions. Since no extraclinical (e.g. biological) indexes of putative nosological discontinuities are available, ${ }^{4}$ our taxonomy is necessarily based on psychopathologically defined syndromes. Hence, psychopathology has become the main method of linking symptoms and diagnosis, but if psychopathology is conflated with nosography, only those symptoms that are supposed to have diagnostic value will be investigated, in a sort of nosographyfocused twilight state where we wear clinical blinkers structured by contemporary classification systems. The focus on diagnosis discourages attention to real people's experiences. As a consequence, clinical utility is confined to ad hoc bits of information useful for clinical decision-making.

\section{Why is psychopathology useful for psychiatry}

There are at least six reasons for psychopathology to be at the heart of psychiatry.

(a) Psychiatry is a heterogeneous discipline. Its practitioners approach the 'object' of their discipline from many different angles, for instance neuroscience, dynamic psychology, and sociology, each of which has its own language, methodology, and practice. Psychiatrists therefore need a common ground and a joint language. Psychopathology does not aim to conceptualise mental disorders - as is the case with psychoanalysis or the cognitive sciences. Psychopathology aspires to respect the phenomenon rather than to market a specific, inflexible theory. Psychopathology, in its Jasperian foundation, is at the end of a continuum where theoretical assumptions are minimised and the forms and contents of the patient's experience are prioritised. Thus, psychopathology can be understood as a shared language that allows clinicians with different theoretical backgrounds to understand each other when dealing with mental disorders. 
(b) Psychiatry aims to establish rigorous diagnoses. Psychopathology is still highly useful in fields where the major disorders cannot be neuroscientifically defined as disease entities, but are exclusively syndromes defined according to characterising symptoms, such as abnormal subjective experiences.

(c) Psychiatry is about understanding disturbed human experience, in addition to diagnosing and classifying that experience. Psychopathology functions as a bridge between the human and clinical sciences, providing the basic tools to make sense of mental suffering. Today, there is a risk that clinical blinkering results in clinicians being only able to view the phenomena relevant to diagnosis and classification and excludes the scrutiny of the diverse and varied nature of what is really there in the patient's experience - the essential prerequisite to understanding his or her illness - and closes us off to the discovery of new psychopathological knowledge.

(d) Psychiatry addresses abnormal human subjectivity. Psychopathology attempts to define what is abnormal (rather than taking for granted common-sense views) and to grasp which elements of mental life remain normal in the context of illness.

(e) Psychiatry is about caring for troubled human existence, rather than judging, marginalising, punishing or stigmatising it. Psychopathology connects understanding with caring, and endeavours to establish a methodological as well as ethical framework for this.

(f) Psychiatry looks for a way to connect first-person subjective experience with brain functioning. Psychopathology is about bridging understanding (meaningfulness) and explanation (causality) in research and clinical settings. Phenomenological psychopathology in itself is prior (i.e. antecedent) to any causal accounts addressing sub-personal mechanisms. At least part of the difficulties in establishing a neuroscientific psychiatry may be insufficient knowledge of psychopathology. Basic psychopathological knowledge is a prerequisite for research in explanatory psychopathologies and it can give new impulse to epistemologically sound biological psychiatry.

\section{Phenomenology and psychopathology}

The basic purpose of psychopathology is to empower psychiatrists with a systematic knowledge of the patients' experiences. This issue is primarily addressed by phenomenology, whose main object is human subjectivity. ${ }^{5}$ Phenomenology is the method that stands at the basis of psychopathology. Phenomenological psychopathology is 'open' to an unusual extent, in that it reveals aspects of experience that other approaches tend to overwrite or eclipse with their strong theoretical claims. In this sense, phenomenological psychopathology can be conceived of as psychopathologia prima. It assumes that the primary object of psychiatry is the patient's subjectivity, thus putting all its efforts into focusing on the patient's state of mind as it is experienced and narrated by them. Expressions and behaviours are considered important, but less specific than personal experiences. The form - that is, the mode in which content is given to consciousness (e.g. verbal-acoustic hallucination) - is considered more important than the content itself (the precise theme, e.g. the verbal hallucination being persecuting). In phenomenological psychopathology, a symptom is the expression of a profound modification of the human subjectivity and its form (more than its content) reveals its underlying characteristic. ${ }^{6}$ The form of a symptom, as it emerges from careful psychopathological analysis, is potentially more informative than surface clinical features ${ }^{7}$ in describing a given phenotype, establishing a reliable diagnosis and understanding the 'world' a patient lives in.

\section{Conclusions}

Psychopathology is not one of numerous approaches aiming at conceptualising mental disorders or illuminating their pathogenesis. It is prior to any causal accounts addressing sub-personal mechanisms. Core psychopathological knowledge is presupposed in order to achieve explanatory ambitions. Psychopathology is 'phenomenological' not in the sense that it embraces a given philosophical approach: rather, it is methodologically so. ${ }^{8}$ Although the emphasis on subjectivity and form may look like a theoretical commitment, that commitment is the product of a stance that seeks to respect the phenomenon rather than impose upon it.

Giovanni Stanghellini, MD, Dr Phil h.c., Department of Psychological, Humanistic and Territorial Studies, G. d'Annunzio University, Chieti, Italy and Diego Portales University, Santiago, Chile; Matthew R. Broome, MRCPsych, PhD, Department of Psychiatry, University of Oxford and Oxford Health NHS Foundation Trust, UK

Correspondence: Giovanni Stanghellini, 'G. d'Annunzio' University, Via dei Vestini 31 - 66100 Chieti Scalo, Italy. Email: giostan@libero.it

First received 4 Jan 2014, accepted 17 Jul 2014

\section{References}

1 Jaspers K. General Psychopathology. Johns Hopkins University Press, 1997.

2 Stanghellini G, Fuchs T (eds) One Century of Karl Jaspers' General Psychopathology. Oxford University Press, 2013.

3 Stanghellini G, Fulford KWM, Bolton D. Person-centered psychopathology of schizophrenia. Building on Karl Jaspers' understanding of the patient's attitude towards his illness. Schizophr Bull 2013; 39: 287-94.

4 Maj M. Mental disorders as 'brain diseases' and Jaspers' legacy. World Psychiatry 2013; 12: 1-3.

5 Gallagher S, Zahavi D. The Phenomenological Mind. An Introduction to Philosophy of Mind and Cognitive Science. Routledge, 2008.

6 Parnas J. The core Gestalt of schizophrenia. World Psychiatry 2012; 11: 67-9.

7 Kendler KS. Introduction: Why does psychiatry need philosophy? In Kendler KS, Parnas J (eds) Philosophical Issues in Psychiatry; Explanation, Phenomenology, and Nosology. Johns Hopkins University Press, 2008.

8 Broome MR, Harland R, Owen GS, Stringaris A. The Maudsley Reader in Phenomenological Psychiatry. Cambridge University Press, 2013. 